\title{
TWO SUPER-EARTHS ORBITING THE SOLAR ANALOG HD 41248 ON THE EDGE OF A 7:5 MEAN MOTION RESONANCE
}

\author{
J. S. Jenkins ${ }^{1,2}$, M. TuOmi ${ }^{2,3}$, R. Brasser ${ }^{4}$, O. IvanyuK ${ }^{5}$, and F. Murgas ${ }^{6,7}$ \\ ${ }^{1}$ Departamento de Astronomia, Universidad de Chile, Camino el Observatorio 1515, Las Condes, Santiago, Casilla 36-D, Chile; jjenkins@ das.uchile.cl \\ ${ }^{2}$ Center for Astrophysics, University of Hertfordshire, College Lane Campus, Hatfield, Hertfordshire, AL10 9AB, UK \\ ${ }^{3}$ Department of Physics and Astronomy, Tuorla Observatory, University of Turku, Väisäläntie 20, FI-21500 Piikkiö, Finland \\ ${ }^{4}$ Institute for Astronomy and Astrophysics, Academia Sinica, Taipei 10617, Taiwan \\ ${ }^{5}$ Main Astronomical Observatory of National Academy of Sciences of Ukraine, 27 Zabolotnoho, Kyiv 127 03680, Ukraine \\ ${ }^{6}$ Instituto de Astrofísica de Canarias, Via Lactea, E-38205 La Laguna, Tenerife, Spain \\ ${ }^{7}$ Departamento de Astrofísica, Universidad de La Laguna (ULL), E-38206 La Laguna, Tenerife, Spain \\ Received 2013 February 22; accepted 2013 April 25; published 2013 June 14
}

\begin{abstract}
There are a growing number of multi-planet systems known to be orbiting their host stars with orbital periods that place them in mean motion resonances (MMRs). These systems are generally in first-order resonances and dynamical studies have focused their efforts on understanding the origin and evolution of such dynamically resonant commensurabilities. Here we report the discovery of two super-Earths that are close to a second-order dynamical resonance orbiting the metal-poor $([\mathrm{Fe} / \mathrm{H}]=-0.43 \mathrm{dex})$ and inactive $\mathrm{G} 2 \mathrm{~V}$ star $\mathrm{HD} 41428$. We analyzed $62 \mathrm{HARPS}$ archival radial velocities for this star that, until now, exhibited no evidence for planetary companions. Using our new Bayesian Doppler signal detection algorithm, we find two significant signals in the data, with periods of 18.357 days and 25.648 days, indicating they could be part of a 7:5 second-order MMR. Both semi-amplitudes are below $3 \mathrm{~m} \mathrm{~s}^{-1}$ and the minimum masses of the pair are 12.3 and $8.6 M_{\oplus}$, respectively. Our simulations found that apsidal alignment stabilizes the system, and even though libration of the resonant angles was not seen, the system is affected by the presence of the resonance and could still occupy the 7:5 commensurability, which would be the first planetary configuration in such a dynamical resonance. Given the multitude of low-mass multi-planet systems that will be discovered in the coming years, we expect that more of these second-order resonant configurations will emerge from the data, highlighting the need for a better understanding of the dynamical interactions between forming planetesimals.
\end{abstract}

Key words: planetary systems - stars: fundamental parameters - stars: individual (HD 41248) - stars: rotation

Online-only material: color figures

\section{INTRODUCTION}

Resonances seem to be a common feature in nature when bodies with measurable gravitational fields interact dynamically with one and other. In the solar system, various bodies are found to be in mean motion resonances (MMRs), yet even though such objects can be found to have period ratios that are close to known MMRs; confirmation of the existence of any MMR can only be made by studying the system dynamically to confirm effects such as libration of the resonant angles. Examples include the 2:5 MMR of Jupiter and Saturn (Michtchenko \& Ferraz-Mello 2001) and the 3:2 MMR between Neptune and Pluto (Peale 1976). In fact, a 1:2 MMR between Jupiter and Saturn may have been the driving force behind the current configuration of the solar systems' outer planets (Gomes et al. 2005; Tsiganis et al. 2005; Morbidelli et al. 2005).

Beyond planetary bodies, there are also various moons and asteroids that exhibit MMRs. A famous example of these comes from Jupiter's moons Ganymede, Europa, and Io that form a 1:2:4 resonant set (Peale 1976). Such resonances provide important constraints on the formation and evolution of migrating bodies and allow a window into the dynamics of evolving systems. Therefore, discovering new resonant exoplanetary systems can provide unique constraints on the early evolution of exoplanets, along with a contextual view of the whole ensemble of planet formation and evolution in general.

Currently we have confirmed a number of exoplanetary systems that have pairs or more of planets in some resonant configuration by radial velocities. 2:1 resonances seem to be the most common by-product of giant planet formation (e.g., Marcy et al. 2001). Further resonances have been witnessed, including a Jupiter-moon-like 1:2:4 orbital resonant set (Rivera et al. 2010); however, these systems tend to be first-order resonances.

Desort et al. (2008) reported the discovery of the HD 60532 exoplanetary system that contains a pair of planets in a secondorder MMR. Again, these planets were found to be gas giants with masses above a Jupiter mass and Laskar \& Correia (2009) confirmed that they are indeed in a 3:1 MMR configuration. Recently, Fabrycky et al. (2012) used Kepler transit timing measurements to detect resonant systems including that of Kepler-29, which seems to have two planets orbiting the star locked in a 9:7 MMR. This system does not have radial velocity information to confirm the nature of the objects; however, this could be the first super-Earth planetary system in such a dynamical commensurability. Kepler data also indicate that $3: 1$ MMRs are the most common second-order resonances (Lissauer et al. 2011).

In this work, we present the discovery of a possible new second-order MMR configuration that has never been previously observed for planets. We show that the magnetically inactive G2V star HD 41248 hosts at least two rocky planets that are on the edge of a 7:5 MMR by analyzing extensive archival data from HARPS that previously did not contain any known Doppler signals. In Section 2 we present the data we have used in this study, then in Section 3 we discuss the comparison between this star and the Sun. In Section 4 we present the Keplerian analysis 
of our radial velocities, and in Section 5 we show that the signals are not associated with any activity-related phenomena. Finally, Section 6 presents our dynamical stability tests for this system and we conclude with a discussion of the system in Section 7.

\section{HD 41248 DATA}

All data in this paper were taken from the ESO HARPS Archive, ${ }^{8}$ a community tool that allows users to download fully reduced and analyzed data that have been processed using the HARPS-DRS Version 3.5. The pipeline performs the usual reduction steps for high-resolution echelle spectra, from bias and flat fielding to extraction and wavelength calibration.

A total of 62 velocities for the star HD 41248 were downloaded and used in this analysis as part of our project to discover new rocky planets orbiting nearby Sun-like stars using our novel methodologies. The baseline of observations is close to $7.5 \mathrm{yr}$ (BJD 2452943.85284 to 2455647.57967) and in general a high level of data quality was maintained. The median signal-to-noise ratio $(\mathrm{S} / \mathrm{N})$ for the set is $\sim 100$ at a wavelength of around $6050 \AA$, with a lowest value of 26 and a highest value of 150 . None of the data we downloaded were rejected from our final analysis.

After reduction and extraction of the observation has finished, post-reduction analysis is also performed on the spectra and this consists of cross-correlating each of the echelle orders with a pre-fabricated binary mask to generate an order-per-order crosscorrelation function $(\mathrm{CCF})$, which are then combined using a weighting scheme to produce a single stable mean CCF. This mean CCF is then fit by a Gaussian function and the Gaussian model allows the software to generate a precise and absolute radial velocity measurement.

The values extracted from the ESO Archive DRS processing are also supported by the velocities generated using the HARPS-TERRA software (Anglada-Escudé \& Butler 2012; Anglada-Escudé et al. 2012), which we use as a sanity check for stars like the Sun as the DRS values tend to produce higher precision for these stars, in comparison to cooler $M$ dwarfs where TERRA works better. The final Barycentric Julian Dates, DRS, and TERRA radial velocities, along with DRS and TERRA uncertainties, are shown in Table 1.

\section{HD 41248 VERSUS THE SUN}

The properties of HD 41248 are summarized in Table 2 but some of the most interesting features are highlighted here. First, the star has a spectral type of $\mathrm{G} 2 \mathrm{~V}$ and a $B-V$ color of 0.624 , meaning that it is a solar analog since the solar values are G2V and 0.642, respectively. Rocky planets around such stars can provide direct tests of planet formation mechanisms and architectures of systems orbiting Sun-like stars.

\subsection{Chromospheric Activity}

Low amplitude signals are most easily sought in radial velocity data of the most quiescent and slowly rotating stars. HD 41248 has a $\log R_{\mathrm{HK}}$ activity index of -4.92 dex and a rotational velocity of only $2.4 \pm \mathrm{km} \mathrm{s}^{-1}$, highlighting that this star is an ideal candidate for such studies. The Sun has a $\log R_{\mathrm{HK}}$ activity index of $-4.91 \mathrm{dex}$ and $v \sin i$ of $1.6 \pm 0.3 \mathrm{~km} \mathrm{~s}^{-1}$ (Pavlenko et al. 2012), showing that both these stars have good agreement in their evolutionary properties, too.

Figure 1 shows the distribution of these activity indices and the best-fit model Gaussian to the data. The $\log R_{\mathrm{HK}}$ values were

\footnotetext{
8 http://www.eso.org/wdb/wdb/eso/repro/form
}

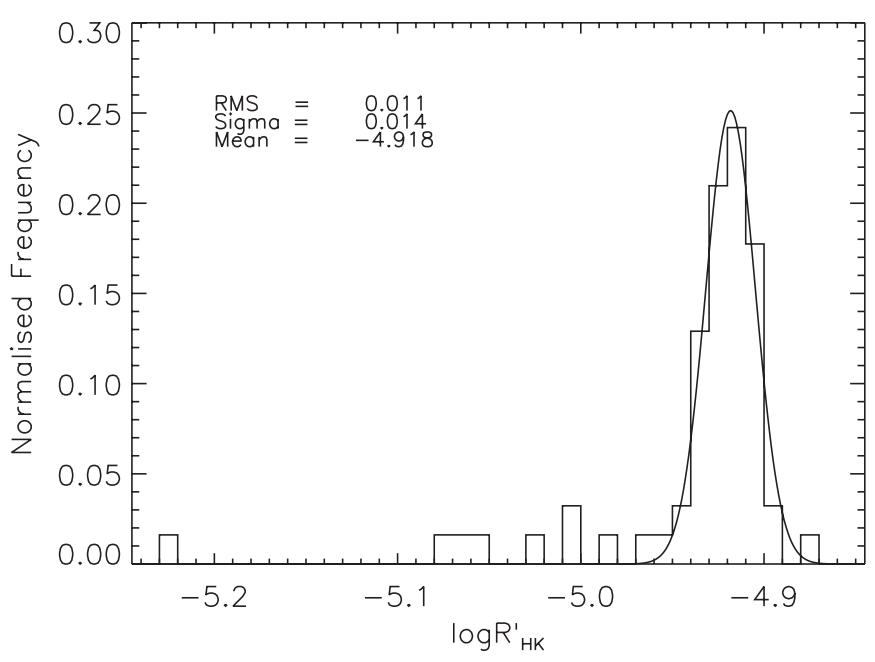

Figure 1. Histogram of the $\log R_{\mathrm{HK}}$ activity indices of the time series data for HD 41248. The best-fit Gaussian model to the activities is represented by the solid curve, and the associated data for this model are shown in the plot.

computed using the HARPS one-dimensional spectra following the procedures explained in Jenkins et al. (2006, 2008, 2011). Clearly, most of the data are tightly clustered around the mean $\log R_{\mathrm{HK}}$ of -4.92 dex. The distribution is tightly packed, with a scatter of only 0.01 dex, which is both the intrinsic variability of the star and the uncertainty in the measurements. There are a few values with activities less than -5 dex; however, these tend to be the lowest $\mathrm{S} / \mathrm{N}$ spectra and therefore there is a bias in the line core measurements as random noise causes negative read values that artificially draw the line core values lower than they really are, and therefore the overall activity index is found to be lower than it should be.

\subsection{Abundance Pattern}

One parameter where there is some difference between the solar value and that of HD 41248 is the stellar metallicity. The iron abundance $([\mathrm{Fe} / \mathrm{H}])$ of this star is $-0.43 \pm 0.10 \mathrm{dex}$, which is significantly lower than the solar value, defined as 0.0 dex. In fact, this low abundance value could be one of the important parameters that differentiates a low-mass, rockydominated system from a gas-giant-dominated system, since the rising power-law probability function that shows that the most metal-rich Sun-like stars have a higher probability of hosting planets (Fischer \& Valenti 2005; Sousa et al. 2011) may in fact not hold true for the rocky super-Earth population (Buchhave et al. 2012; Jenkins et al. 2013). Therefore, HD 41248 can be classed as a metal-deficient and old solar analog.

Table 3 lists various abundances for volatile elements in the atmosphere of HD 41248. We measured these values directly from the spectra using the method explained in Pavlenko et al. (2012) and more details of how we arrived at these values will be discussed in O. Ivanyuk et al. (2013, in preparation). These abundances generally track the low $[\mathrm{Fe} / \mathrm{H}]$ values, and in comparison to the Sun, all of the elements we have considered here are depleted. Therefore, we expect that the nascent disk from which planets could form was metal-deficient, are hence depleted in the typical elements we expect are processed into planetesimals to form systems of planets through core accretion.

Finally, it is necessary to know the stellar mass of HD 41248 so that we have a handle on the masses of any planets detected orbiting the star from the Doppler curve. Given the $T_{\text {eff }}$ we measure of $5713 \pm 50 \mathrm{~K}$, the absolute magnitude $\left(M_{V}\right)$ of 
Table 1

HARPS Radial Velocities for HD 41248

\begin{tabular}{|c|c|c|c|c|c|c|c|c|}
\hline $\begin{array}{l}\text { BJD } \\
\text { (days) }\end{array}$ & $\begin{array}{c}\text { DRS-RV } \\
\left(\mathrm{ms}^{-1}\right)\end{array}$ & $\begin{array}{c}\text { DRS- } \sigma_{\mathrm{RV}} \\
\left(\mathrm{ms}^{-1}\right)\end{array}$ & $\begin{array}{c}\text { TERRA-RV } \\
\left(\mathrm{ms}^{-1}\right)\end{array}$ & $\begin{array}{c}\text { TERRA- } \sigma_{\mathrm{RV}} \\
\left(\mathrm{ms}^{-1}\right)\end{array}$ & $\begin{array}{l}S_{\mathrm{MW}} \\
(\mathrm{dex})\end{array}$ & $\begin{array}{l}\log R_{\mathrm{HK}} \\
\quad(\mathrm{dex})\end{array}$ & $\begin{array}{c}\text { BIS } \\
\left(\mathrm{ms}^{-1}\right)\end{array}$ & $\begin{array}{l}\text { FWHM } \\
\left(\mathrm{ms}^{-1}\right)\end{array}$ \\
\hline 2452943.8528426 & 3526.591 & 2.588 & -3.512 & 2.591 & $0.1686 \pm 0.0024$ & -4.9348 & $35.92 \pm 3.66$ & 6721.78 \\
\hline 2452989.7102293 & 3519.139 & 4.063 & -7.736 & 4.223 & $0.1566 \pm 0.0045$ & -5.0089 & $27.39 \pm 5.74$ & 6719.01 \\
\hline 2452998.6898180 & 3526.427 & 5.425 & 1.179 & 6.226 & $0.1495 \pm 0.0062$ & -5.0599 & $33.53 \pm 7.67$ & 6701.20 \\
\hline 2453007.6786518 & 3526.626 & 2.525 & -3.456 & 2.232 & $0.1609 \pm 0.0025$ & -4.9810 & $28.61 \pm 3.57$ & 6718.19 \\
\hline 2453787.6079555 & 3522.442 & 2.758 & -3.192 & 3.353 & $0.1566 \pm 0.0031$ & -5.0091 & $31.30 \pm 3.90$ & 6718.53 \\
\hline 2454055.8375443 & 3523.175 & 2.062 & -6.636 & 2.226 & $0.1674 \pm 0.0018$ & -4.9418 & $23.94 \pm 2.91$ & 6714.51 \\
\hline 2454789.7207967 & 3522.987 & 0.817 & -4.361 & 0.897 & $0.1705 \pm 0.0005$ & -4.9241 & $27.43 \pm 1.15$ & 6722.19 \\
\hline 2454790.6943362 & 3519.487 & 0.899 & -6.817 & 0.916 & $0.1698 \pm 0.0005$ & -4.9281 & $30.82 \pm 1.27$ & 6724.20 \\
\hline 2454791.7055725 & 3522.466 & 0.834 & -4.765 & 0.831 & $0.1708 \pm 0.0005$ & -4.9225 & $29.54 \pm 1.18$ & 6720.59 \\
\hline 2454792.7042506 & 3522.290 & 0.795 & -4.763 & 0.779 & $0.1723 \pm 0.0005$ & -4.9143 & $28.09 \pm 1.12$ & 6728.64 \\
\hline 2454793.7211230 & 3524.992 & 0.890 & -2.854 & 0.984 & $0.1727 \pm 0.0005$ & -4.9124 & $25.27 \pm 1.25$ & 6727.73 \\
\hline 2454794.6946036 & 3527.038 & 0.893 & 0.860 & 1.051 & $0.1715 \pm 0.0005$ & -4.9191 & $29.58 \pm 1.26$ & 6732.04 \\
\hline 2454795.7156306 & 3528.445 & 0.913 & 0.874 & 0.909 & $0.1737 \pm 0.0005$ & -4.9070 & $29.54 \pm 1.29$ & 6725.91 \\
\hline 2454796.7195391 & 3528.207 & 0.957 & 0.513 & 0.975 & $0.1736 \pm 0.0006$ & -4.9076 & $30.32 \pm 1.35$ & 6727.87 \\
\hline 2454797.7051254 & 3528.994 & 0.908 & 3.036 & 1.137 & $0.1750 \pm 0.0005$ & -4.9001 & $27.76 \pm 1.28$ & 6733.21 \\
\hline 2454798.6972277 & 3531.198 & 0.915 & 3.831 & 0.906 & $0.1731 \pm 0.0005$ & -4.9101 & $25.13 \pm 1.29$ & 6731.22 \\
\hline 2454902.5907553 & 3525.084 & 2.021 & 0.000 & 2.525 & $0.1796 \pm 0.0018$ & -4.8768 & $21.34 \pm 2.85$ & 6729.22 \\
\hline 2454903.5172666 & 3527.587 & 0.783 & 0.359 & 0.909 & $0.1722 \pm 0.0004$ & -4.9151 & $27.68 \pm 1.10$ & 6726.66 \\
\hline 2454904.5185682 & 3525.760 & 0.901 & -2.032 & 0.862 & $0.1737 \pm 0.0005$ & -4.9071 & $27.18 \pm 1.27$ & 6722.67 \\
\hline 2454905.5355291 & 3527.552 & 0.898 & -0.148 & 0.897 & $0.1706 \pm 0.0005$ & -4.9240 & $27.97 \pm 1.27$ & 6722.94 \\
\hline 2454906.5179999 & 3527.947 & 1.041 & 0.122 & 1.114 & $0.1713 \pm 0.0006$ & -4.9201 & $31.02 \pm 1.47$ & 6723.85 \\
\hline 2454907.5647983 & 3527.517 & 0.905 & 0.140 & 1.123 & $0.1731 \pm 0.0006$ & -4.9101 & $28.88 \pm 1.28$ & 6727.04 \\
\hline 2454908.5603822 & 3526.226 & 0.804 & -2.046 & 0.961 & $0.1721 \pm 0.0004$ & -4.9155 & $27.94 \pm 1.13$ & 6720.84 \\
\hline 2454909.5380036 & 3527.107 & 0.854 & -0.513 & 0.928 & $0.1699 \pm 0.0005$ & -4.9277 & $25.45 \pm 1.20$ & 6721.35 \\
\hline 2454910.5385064 & 3528.236 & 1.115 & 1.119 & 1.170 & $0.1727 \pm 0.0008$ & -4.9121 & $27.50 \pm 1.57$ & 6726.82 \\
\hline 2454911.5427244 & 3524.975 & 0.747 & -3.570 & 0.722 & $0.1715 \pm 0.0004$ & -4.9189 & $28.13 \pm 1.05$ & 6721.04 \\
\hline 2454912.5392921 & 3525.216 & 0.739 & -3.038 & 0.898 & $0.1719 \pm 0.0004$ & -4.9167 & $25.78 \pm 1.04$ & 6718.78 \\
\hline 2455284.5272133 & 3528.228 & 0.734 & -1.092 & 0.917 & $0.1751 \pm 0.0004$ & -4.8998 & $28.42 \pm 1.03$ & 6724.46 \\
\hline 2455287.5109103 & 3524.603 & 0.881 & -4.445 & 0.921 & $0.1743 \pm 0.0005$ & -4.9038 & $25.18 \pm 1.24$ & 6733.01 \\
\hline 2455288.5285775 & 3523.316 & 0.754 & -4.407 & 0.807 & $0.1731 \pm 0.0004$ & -4.9104 & $25.43 \pm 1.06$ & 6730.40 \\
\hline 2455289.5460248 & 3526.698 & 0.789 & -1.501 & 0.923 & $0.1743 \pm 0.0004$ & -4.9036 & $26.29 \pm 1.11$ & 6723.59 \\
\hline 2455290.5095380 & 3525.900 & 0.891 & -0.647 & 0.996 & $0.1737 \pm 0.0005$ & -4.9068 & $21.95 \pm 1.26$ & 6727.32 \\
\hline 2455291.5216615 & 3526.083 & 1.006 & -3.054 & 1.060 & $0.1707 \pm 0.0006$ & -4.9233 & $26.85 \pm 1.42$ & 6734.67 \\
\hline 2455293.5043818 & 3527.359 & 0.974 & 1.254 & 1.113 & $0.1719 \pm 0.0006$ & -4.9165 & $27.39 \pm 1.37$ & 6727.22 \\
\hline 2455304.5180173 & 3522.412 & 1.689 & -0.532 & 5.028 & $0.1318 \pm 0.0038$ & -5.2203 & $31.20 \pm 2.38$ & 6801.22 \\
\hline 2455328.4550220 & 3529.109 & 0.791 & 2.304 & 0.828 & $0.1746 \pm 0.0005$ & -4.9022 & $20.73 \pm 1.11$ & 6735.38 \\
\hline 2455334.4564390 & 3532.003 & 1.365 & 7.425 & 1.432 & $0.1685 \pm 0.0014$ & -4.9353 & $25.33 \pm 1.93$ & 6737.32 \\
\hline 2455387.9305071 & 3530.983 & 1.039 & 4.143 & 0.986 & $0.1708 \pm 0.0008$ & -4.9225 & $27.29 \pm 1.47$ & 6737.65 \\
\hline 2455390.9312188 & 3531.330 & 1.577 & 4.830 & 1.517 & $0.1635 \pm 0.0013$ & -4.9652 & $30.24 \pm 2.23$ & 6734.97 \\
\hline 2455434.8790630 & 3516.749 & 3.334 & -4.089 & 3.355 & $0.1479 \pm 0.0036$ & -5.0724 & $31.59 \pm 4.71$ & 6740.53 \\
\hline 2455439.8843407 & 3527.755 & 3.258 & 10.200 & 4.319 & $0.1488 \pm 0.0038$ & -5.0649 & $32.80 \pm 4.60$ & 6723.47 \\
\hline 2455445.9241644 & 3522.932 & 3.113 & -0.217 & 3.839 & $0.1545 \pm 0.0036$ & -5.0233 & $28.30 \pm 4.40$ & 6727.79 \\
\hline 2455465.8566225 & 3526.615 & 1.412 & 1.415 & 1.440 & $0.1706 \pm 0.0012$ & -4.9237 & $23.18 \pm 1.99$ & 6731.85 \\
\hline 2455480.8795598 & 3527.592 & 1.133 & 1.534 & 1.245 & $0.1706 \pm 0.0008$ & -4.9237 & $27.09 \pm 1.60$ & 6728.47 \\
\hline 2455483.8136024 & 3525.010 & 1.740 & -1.948 & 1.837 & $0.1704 \pm 0.0015$ & -4.9250 & $27.97 \pm 2.46$ & 6734.01 \\
\hline 2455488.8262312 & 3525.839 & 0.747 & -0.835 & 0.818 & $0.1708 \pm 0.0004$ & -4.9226 & $31.07 \pm 1.05$ & 6725.47 \\
\hline 2455494.8532009 & 3529.808 & 0.954 & 3.114 & 1.123 & $0.1686 \pm 0.0006$ & -4.9348 & $26.88 \pm 1.34$ & 6730.94 \\
\hline 2455513.7823100 & 3528.860 & 1.286 & -0.462 & 1.438 & $0.1715 \pm 0.0009$ & -4.9186 & $35.60 \pm 1.81$ & 6740.83 \\
\hline 2455516.7515789 & 3530.744 & 0.936 & 4.112 & 1.032 & $0.1749 \pm 0.0007$ & -4.9005 & $22.71 \pm 1.32$ & 6736.78 \\
\hline 2455519.7046533 & 3528.854 & 1.198 & 3.453 & 1.116 & $0.1682 \pm 0.0009$ & -4.9375 & $21.95 \pm 1.69$ & 6735.81 \\
\hline 2455537.7997291 & 3527.714 & 0.718 & 0.897 & 0.859 & $0.1756 \pm 0.0005$ & -4.8968 & $27.01 \pm 1.01$ & 6730.29 \\
\hline 2455545.7213656 & 3529.198 & 0.854 & 3.099 & 0.990 & $0.1749 \pm 0.0005$ & -4.9006 & $26.68 \pm 1.20$ & 6737.59 \\
\hline 2455549.7548559 & 3527.949 & 0.833 & -0.567 & 0.896 & $0.1714 \pm 0.0005$ & -4.9195 & $31.97 \pm 1.17$ & 6734.54 \\
\hline 2455576.7923481 & 3525.346 & 1.014 & -1.481 & 1.120 & $0.1654 \pm 0.0007$ & -4.9533 & $29.17 \pm 1.43$ & 6733.68 \\
\hline 2455580.7312518 & 3519.411 & 0.895 & -7.373 & 0.965 & $0.1688 \pm 0.0006$ & -4.9339 & $32.94 \pm 1.26$ & 6729.42 \\
\hline 2455589.7734088 & 3528.423 & 1.420 & 0.958 & 1.579 & $0.1684 \pm 0.0013$ & -4.9360 & $24.59 \pm 2.00$ & 6733.73 \\
\hline 2455612.6068850 & 3527.663 & 0.816 & 0.976 & 0.713 & $0.1714 \pm 0.0005$ & -4.9193 & $25.58 \pm 1.15$ & 6731.09 \\
\hline 2455623.6361828 & 3528.301 & 1.175 & 0.455 & 1.316 & $0.1685 \pm 0.0011$ & -4.9357 & $28.96 \pm 1.66$ & 6726.23 \\
\hline 2455629.5528393 & 3528.652 & 0.944 & 1.864 & 1.002 & $0.1682 \pm 0.0006$ & -4.9370 & $25.71 \pm 1.33$ & 6732.34 \\
\hline 2455641.5542106 & 3528.979 & 1.005 & 1.879 & 1.012 & $0.1697 \pm 0.0007$ & -4.9286 & $28.91 \pm 1.42$ & 6727.20 \\
\hline 2455644.5845033 & 3529.351 & 1.260 & 3.245 & 1.381 & $0.1742 \pm 0.0006$ & -4.9043 & $19.09 \pm 1.78$ & 6740.10 \\
\hline 2455647.5796694 & 3529.945 & 1.372 & 1.898 & 1.259 & $0.1669 \pm 0.0012$ & -4.9448 & $29.10 \pm 1.94$ & 6737.56 \\
\hline
\end{tabular}


Table 2

Stellar Parameters for HD 41248

\begin{tabular}{|c|c|c|}
\hline Parameter & HD 41248 & Reference \\
\hline R.A. J2000 (h:m:s) & $06^{\mathrm{h}} 00^{\mathrm{m}} 32^{\mathrm{s}} .781$ & Perryman et al. (1997) \\
\hline Decl. J2000 (d:m:s) & $-56^{\circ} 09^{\prime} 42^{\prime \prime} 61$ & Perryman et al. (1997) \\
\hline Spectral type & $\mathrm{G} 2 \mathrm{~V}$ & Perryman et al. (1997) \\
\hline$B-V$ & 0.624 & Perryman et al. (1997) \\
\hline$V$ & 8.82 & Perryman et al. (1997) \\
\hline Distance (pc) & $52.38 \pm 1.95$ & van Leeuwen (2007) \\
\hline$M_{V}$ & $5.22 \pm 0.08$ & This work \\
\hline $\log R_{\mathrm{HK}}^{\prime}$ & -4.94 & This work \\
\hline Hipparcos $N_{\mathrm{obs}}$ & 106 & Perryman et al. (1997) \\
\hline Hipparcos $\sigma$ & 0.015 & Perryman et al. (1997) \\
\hline$\Delta M_{V}$ & -0.700 & Jenkins et al. (2011) \\
\hline$L_{\star} / L_{\odot}$ & $0.68 \pm 0.03$ & This Work \\
\hline$M_{\star} / M_{\odot}$ & $0.92 \pm 0.05$ & This Work \\
\hline$R_{\star} / R_{\odot}$ & $0.78 \pm 0.04$ & This Work \\
\hline$T_{\text {eff }}(\mathrm{K})$ & $5713 \pm 50$ & O. Ivanyuk et al. (2013, in preparation) \\
\hline$[\mathrm{Fe} / \mathrm{H}]$ & $-0.43 \pm 0.10$ & O. Ivanyuk et al. (2013, in preparation) \\
\hline $\log g$ & $4.48 \pm 0.10$ & O. Ivanyuk et al. (2013, in preparation) \\
\hline$U, V, W\left(\mathrm{~km} \mathrm{~s}^{-1}\right)$ & $-12.97,-17.81,26.74$ & Jenkins et al. (2011) \\
\hline$P_{\mathrm{rot}, v \sin i}$ (days) & 16 & This Work \\
\hline$v \sin i\left(\mathrm{~km} \mathrm{~s}^{-1}\right)$ & $2.4 \pm 0.2$ & O. Ivanyuk et al. (2013, in preparation) \\
\hline Age (Gyr) & $2_{-2}^{+3}$ & This Work \\
\hline Jitter fit $\left(\mathrm{m} \mathrm{s}^{-1}\right)$ & $0.90_{-0.33}^{+0.94}$ & This Work \\
\hline
\end{tabular}

Table 3

Chemical Abundances for HD 41248

\begin{tabular}{lccc}
\hline \hline Element & $\begin{array}{c}{[\mathrm{X} / \mathrm{H}]} \\
(\mathrm{dex})\end{array}$ & $\begin{array}{c}{[\mathrm{X} / \mathrm{H}]_{\odot}} \\
(\mathrm{dex})\end{array}$ & $\begin{array}{c}{[\mathrm{X} / \mathrm{Fe}]} \\
(\mathrm{dex})\end{array}$ \\
\hline Si I & $-4.743 \pm 0.031$ & -0.34 & 0.09 \\
Si II & $-4.595 \pm 0.025$ & -0.20 & $0.24^{\mathrm{a}}$ \\
Ca I & $-5.972 \pm 0.017$ & -0.31 & -1.14 \\
Ti I & $-7.419 \pm 0.017$ & -0.44 & $-2.59^{\mathrm{b}}$ \\
Ti II & $-7.326 \pm 0.032$ & -0.35 & -2.49 \\
V I & $-8.348 \pm 0.216$ & -0.31 & $-3.52^{\mathrm{a}}$ \\
Cr I & $-6.791 \pm 0.020$ & -0.35 & -1.96 \\
Fe I & $-4.833 \pm 0.007$ & -0.42 & $\ldots$ \\
Fe II & $-4.845 \pm 0.018$ & -0.44 & -0.02 \\
Ni I & $-6.206 \pm 0.010$ & -0.39 & -1.37 \\
\hline
\end{tabular}

Notes.

a Only a few absorption lines used in the analysis.

b Significant non-LTE effects.

$5.22 \pm 0.08$ mags, and the metallicity of $-0.43 \pm 0.1 \mathrm{dex}$, we find a stellar mass of $0.92 \pm 0.05 M_{\odot}$. This is in agreement with other works that find similar values, both lower $\left(0.81 \mathrm{M}_{\odot}\right.$; Sousa et al. 2011) and higher (0.97; Casagrande et al. 2011).

\section{DOPPLER ANALYSIS}

The radial velocity time series of 62 observations for HD 41248 show evidence for at least two low-amplitude signals embedded in the data. Both a periodogram analysis and our Bayesian fitting method detected these signals; however, the Bayesian method can detect the second signal with a high degree of significance such that we can confirm the signal is robust.

\subsection{Periodogram Analysis}

A periodogram search for strong and stable frequencies in the radial velocity data set for HD 41248 reveals a significant peak around 18 days. The top panel of Figure 2 shows these frequencies, with the horizontal dashed line marking the $1 \%$ false alarm probability (FAP) and the horizontal dot-dashed line marking the $0.1 \%$ FAP, both of which were measured using a bootstrap analysis technique (see Anglada-Escudé et al. 2012). It can be seen that the signal is stronger than both these boundaries.

The FAPs from the bootstrap analysis allow us to understand the significance of the frequency peaks we detect in the data. Bootstrapping is appropriate in this case since we can generate test data sets from the original data set without assuming any underlying distribution for the velocities or, more importantly, their uncertainties. Currently, a great amount of effort is being exerted to understand how the combination of white and red noise affects the overall uncertainty we can assume for any radial velocity time series, particulary in the HARPS data (Baluev 2013; Tuomi et al. 2013b); however, it is still an extremely difficult task to model such data with any high degree of accuracy.

We resampled the HD 41248 data 10,000 times with replacement, using a Monte Carlo approach to scramble the timestamps of the velocities, but retaining the velocities and their associated uncertainties. With each new sample, we recompute the Lomb-Scargle (LS) periodogram and measure the strength of the strongest peak that we find. The strength of this strongest peak is then compared to the strength of the original peak from the observed data set and the FAP relates to the number of times a peak stronger than this one is found. In this way we can directly measure the probability from the data in an unparametric way.

The center panel in Figure 2 shows the periodogram of the residuals to the best-fit Keplerian of $\sim 18$ days. There is a clear emerging signal around 25 days, indicating that another physical process is causing a frequency peak at this period; however, the significance is below the $1 \%$ FAP level, meaning that the archival data we have used are not yet abundant enough to confirm the nature of this peak.

The lower plot in Figure 2 shows the periodogram power for the widow function, with the strongest energies found at long periods, beyond the baseline of the data. No sampling 

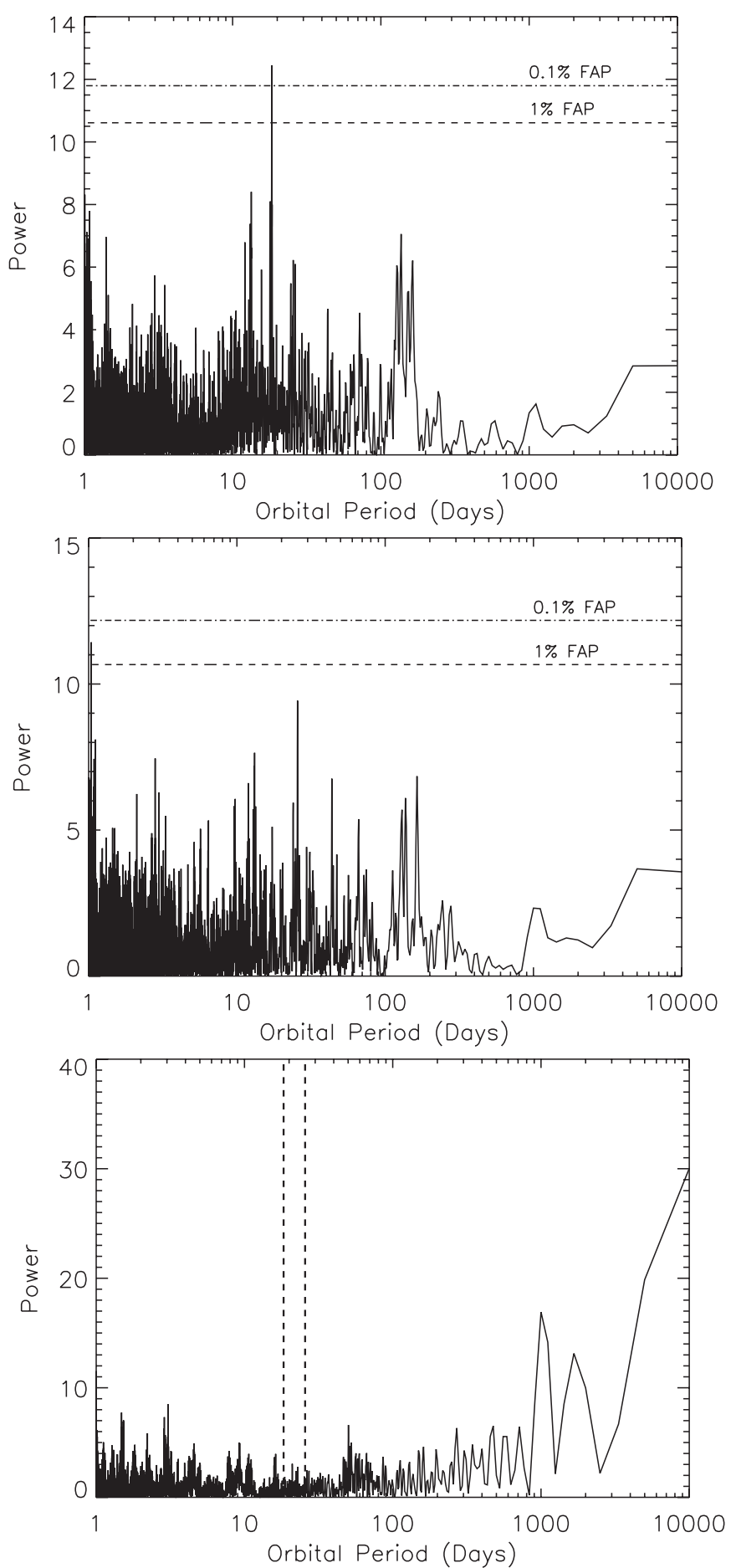

Figure 2. Lomb-Scargle periodograms of the HD 41248 radial velocity data set are shown in the top plot with the same analysis performed on the residuals to the one-planet fit shown in the lower plot. The dashed and dot-dashed lines mark the $1 \%$ and $0.1 \%$ FAP limits. The lower plot shows the window function along with two vertical dashed lines that show the positions of the two signals.

power is found around 18 or 25 days, indicating that the periods we detect in the radial velocities are not sampling features. Also, no sampling power was found around 65 days, which is an alias that could arise since $1 / 64.8 \approx 1 / 18.4-1 / 25.7$; however, we do find some activity power around 60-70 days which we discuss later, but our Bayesian analysis indicates this is not the source of the secondary signal and it is a real Doppler shift, not an alias. Such a tantalizing system necessitates another methodology to confirm the significance of this signal and we turn to our Bayesian analysis method since this has been shown to efficiently detect significant signals with less velocity-rich data than can be accomplished using a standard LS periodogram analysis (Tuomi \& Jenkins 2012).

\subsection{Bayesian Search}

Bayesian signal detection techniques have recently been introduced to spearhead the detection of radial velocity signatures of low-mass exoplanets around nearby stars (e.g., AngladaEscudé \& Tuomi 2012; Tuomi \& Jones 2012; Tuomi et al. 2013 b), yet they have been shown to be rather immune to detections of false positives (e.g., Tuomi 2011). We analyzed the HD 41248 velocities using posterior sampling techniques and a Bayesian model selection to find the best statistical descriptions, i.e., models, of the data, and to obtain estimates for the parameter probability densities of the corresponding models. Following Tuomi \& Jones (2012), we performed the samplings using the adaptive Metropolis algorithm (Haario et al. 2001) and calculated the Bayesian evidence of each model using their truncated posterior mixture estimates. As we analyzed the data in the Bayesian context, we defined the prior probability densities and model probabilities according to the choices of Tuomi (2012) and Tuomi et al. (2013b).

When modeling the data, we used a common Gaussian whitenoise model as a reference model and attempted to improve this description by including correlations within it. This means that the measurement mean is described using a function $\mu(\theta, \xi, t)$, where $\theta$ is the parameter vector, $t$ represents time, and $\xi$ is a vector containing any other variables that might have an effect on the function. With this notation, we defined this model as

$$
\mu(\theta, \xi, t)=f_{k}\left(\theta_{p}, t\right)+\gamma+\sum_{i=1}^{p} c_{i} \xi_{i}
$$

where $f_{k}(t)$ is a function describing the superposition of $k$ Keplerian curves with orbital parameters $\theta_{p}, \gamma$ is a reference velocity with respect to the data mean, and the parameters $c_{i}$ describe the linear dependence of the function on the variables $\xi_{i}$. We used the $S$ index, BIS, and FWHM as these variables to be able to take into account the correlations of the radial velocities with these activity-related indices.

At this point, we can introduce the activity indicators that we use in our Bayesian model. The BIS values and the FWHMs are taken directly from the HARPS-DRS output and details of their origin and usefulness can be found in Queloz et al. (2001) and Santos et al. (2010). The chromospheric $S$ indices have been measured following our own recipes, as mentioned in Section 3. Using this procedure, the uncertainties of these HARPS $S$ indices are found to be less than $1 \%$.

Our results indicate that there are two significant periodicities in the HD 41248 velocities at 18.4 and 25.6 days. We demonstrate the significance of the two signals by showing the logBayesian evidence of models with up to two Keplerian signals (Table 4). Accounting for the correlations between the velocities and the activity indices improves the model clearly for $k=0$ and $k=1$, but only marginally for $k=2$. However, the two-Keplerian model is clearly the best description of the data regardless of whether or not we account for these correlations and the second signal is detected according to the detection criteria of Tuomi (2012) because the two-Keplerian model is $3.3 \times 10^{3}$ times more probable than the one-Keplerian model. The other two detection criteria, i.e., that the orbital periods and 

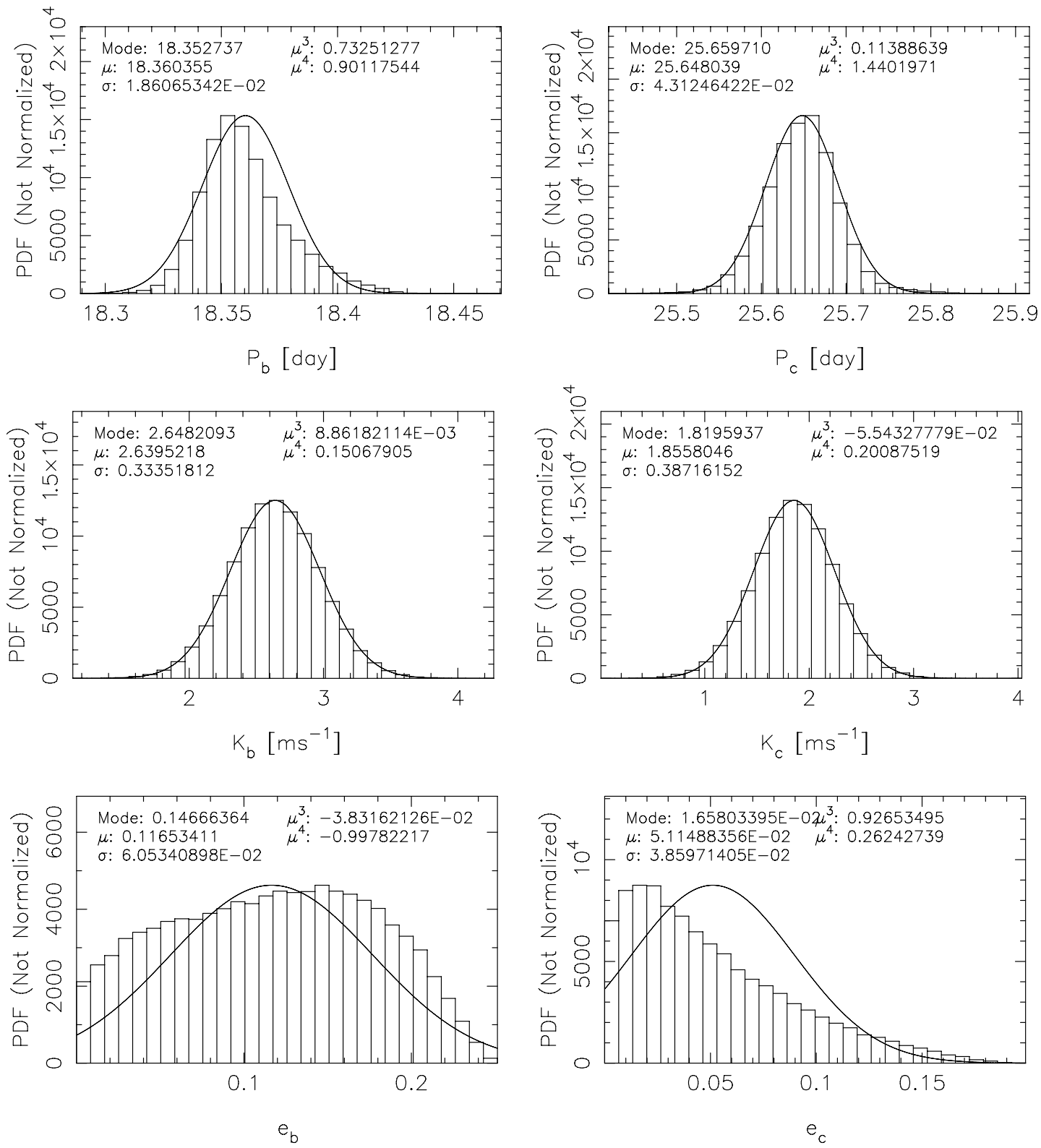

Figure 3. Left column corresponds to the short period signal and the right column corresponds to the longer period signal. The top two histograms show the estimated probability density function of periods from the Bayesian analysis, the middle histograms are the same for the semi-amplitude of the signals, and the lower two histograms are for the eccentricities of the signals. Also overplotted by the solid curves are Gaussians with the same mean and sigma as the distributions.

Table 4

Model Probabilities

\begin{tabular}{ccccc}
\hline \hline$k$ & $\begin{array}{c}\ln \mathrm{BE} \\
(\mathrm{Ref})\end{array}$ & $\begin{array}{c}\text { ln BE } \\
(\text { Cor })\end{array}$ & $\begin{array}{c}P(k) \\
(\text { Ref })\end{array}$ & $\begin{array}{c}P(k) \\
(\text { Cor })\end{array}$ \\
\hline 0 & -158.1 & -154.9 & $5.3 \times 10^{-13}$ & $6.0 \times 10^{-12}$ \\
1 & -141.5 & -136.5 & $4.5 \times 10^{-6}$ & $3.0 \times 10^{-4}$ \\
2 & -128.5 & -127.7 & $\sim 1$ & $\sim 1$ \\
\hline
\end{tabular}

Notes. Log-Bayesian evidence and associated probabilities for the Keplerian models from $k=0, \ldots, 2$ for the HD 41248 velocities taking into account the correlation terms with activity indices (Cor) and without including these terms (Ref).

radial velocity amplitudes are well constrained, are also satisfied. We demonstrate this by plotting the estimated posterior densities of the orbital periods, velocity amplitudes, and orbital eccentricities in Figure 3. We have also tabulated our estimates for model parameters in Table 5 .
Table 5

Keplerian Solutions for HD 41248

\begin{tabular}{lcc}
\hline \hline Parameter & HD $41248 b$ & HD 41248 $c$ \\
\hline$P($ days $)$ & $18.357[18.313,18.423]$ & $25.648[25.518,25.768]$ \\
$e$ & $0.15[0,0.26]$ & $0.00[0,0.18]$ \\
$\omega(\mathrm{rad})$ & $3.2[0,2 \pi]$ & $1.0[0,2 \pi]^{1}$ \\
$M_{0}(\mathrm{rad})$ & $0.2[0,2 \pi]$ & $1.7[0,2 \pi]^{1}$ \\
$K\left(\mathrm{~ms}^{-1}\right)$ & $2.93[1.65,3.65]$ & $1.84[0.67,2.97]$ \\
$a(\mathrm{AU})$ & $0.137[0.126,0.154]$ & $0.172[0.158,0.192]$ \\
$m_{p} \sin i\left(M_{\oplus}\right)$ & $12.3[6.9,16.5]$ & $8.6[3.6,15.1]$ \\
\hline$\gamma\left(\mathrm{ms}^{-1}\right)$ & $-48[-1.36,0.18]$ & $\ldots$ \\
$\sigma_{J}\left(\mathrm{~ms}^{-1}\right)$ & $0.90[0.57,1.84]$ & $\cdots$ \\
$c_{1}\left(\mathrm{~ms}^{-1} \mathrm{dex}^{-1}\right)$ & $147[-20,296]$ & $\ldots$ \\
$c_{2}$ & $0.000[-0.232,0.229]$ & $\cdots$ \\
$c_{3}\left(10^{-3}\right)$ & $61[-14,156]$ & $\cdots$ \\
\hline
\end{tabular}

Notes. The maximum a posteriori estimates of the solution for HD 41248 velocities and the $99 \%$ Bayesian credibility intervals from posterior samplings. 
Obtaining the samples from the posterior densities was simple in this case because the period space contained only two significant maxima corresponding to the two signals we observed. Despite several attempts, we could not find a third signal and the samplings of the parameter space of a threeKeplerian model did not converge to a third periodicity. We also attempted to include a moving average (MA) component in the statistical model to improve its performance and to take into account intrinsic correlations in the measurement noise (Tuomi et al. 2013a, 2013b). However, the corresponding parameter describing the amount of autocorrelation in the data was found to be consistent with negligible estimates, and the corresponding MA component did not improve the statistical model. Furthermore, as can be seen in Table 5, all the parameters quantifying the linear dependence of the velocities on the different activity indices $\left(c_{1}, c_{2}\right.$, and $\left.c_{3}\right)$ are consistent with zero (i.e., cannot be shown different from zero with a $99 \%$ credibility), which explains why taking these correlations into account improved the statistical model little in terms of Bayesian evidences (Table 4). However, there are likely correlations between the velocities and $S$ index and FWHM, but BIS cannot be shown to be correlated with them at all.

Based on the Bayesian analyses of the HD 41248 radial velocities, there are two significant periodicities in the data corresponding to two super-Earths or Neptune-mass planets on nearby close-in orbits (Figure 4). In fact, their orbits are so close to one another that the $99 \%$ credibility intervals of $a_{b}$ and $a_{c}$ taking into account the uncertainty in the stellar mass are almost overlapping (Table 5) and the configuration cannot be immediately stated to be stable in the long term. However, orbital stability is suggested by the fact that the ratio of the orbital periods is almost exactly 1.4 with a Bayesian $99 \%$ interval of $[1.384,1.407]$, which suggests a possible $7: 5$ MMR. The eccentricities of the system also show an interesting configuration with the inner planet having an eccentricity around 0.2 and the outer planet having an eccentricity close to circular. In fact, we tested if the inner planet's eccentricity was closer to zero by changing the eccentricity prior model to favor a more circular orbit, but the eccentricity was found to be 0.22 in this case, indicating that the inner planet does have some genuine measurable eccentricity given the data. If we can rule out the source of these signals as originating from stellar magnetic activity, this could be a remarkable 7:5 MMR system, and therefore we assess this possibility in Section 6 by analyzing the stability of the system.

\section{ACTIVITY INDICATORS}

Within our Bayesian model we have taken into account any linear correlations between the radial velocities and the activity indicators (BIS, FWHM, and $S_{\text {HARPS }}$ ), as explained in the previous section. However, not only does the presence of activity add additional noise to any individual radial velocity measurement, but it can also mimic the presence of a true Doppler-induced frequency in the full time series of radial velocity data (e.g., Queloz et al. 2001). Therefore, it is necessary to perform a test on whatever activity indicators one has on hand in order to search for periodicities that could be associated with the periodicities found in the velocities.

In Figure 5 we show the periodograms for each of these activity indices, going from the $S$ indices at the top to the BIS values in the center, and finally the FWHMs at the bottom. The $S$ indices show a strong periodogram peak at 61 days and another slightly weaker peak at 69 days which could be the rotational
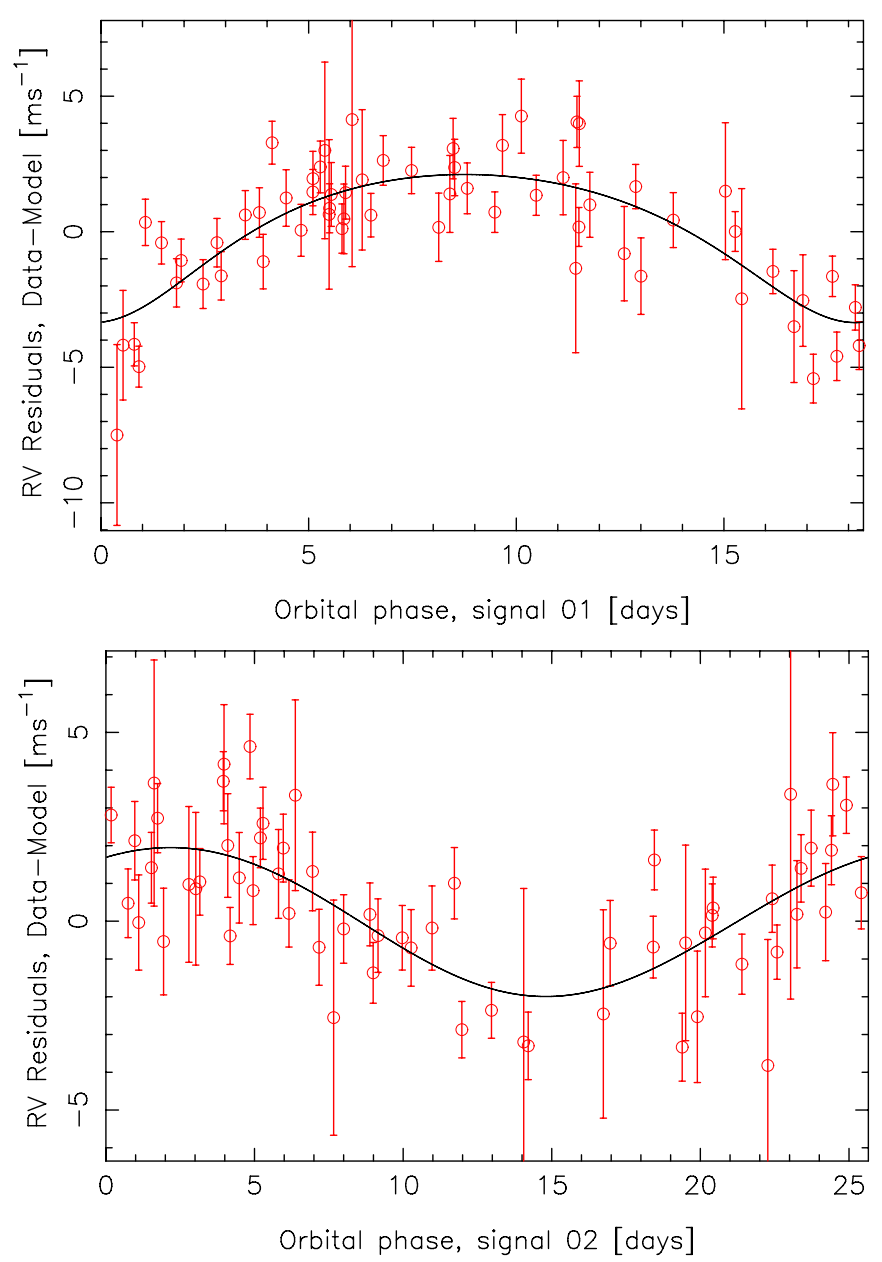

Figure 4. Phased radial velocity curves for planets HD $41248 b$ and $c$ from the Bayesian-detected periods.

(A color version of this figure is available in the online journal.)

period of the star or some other activity cycle. There is no significantly strong frequency peak in the BIS periodogram; however, in the FWHM periodogram there is a strong peak emerging at $\sim 71$ days that could be related to the same frequency detected in the $S$ indices. If so this may support the hypothesis that the rotational period of this star, or a harmonic thereof, is around 70 days, or at least that there is an activity cycle present at that frequency.

The inner signal in the radial velocities at 18 days appears close to a peak in the BIS periodogram at around 18 days and this could shed some doubt on the nature of the inner signal, even though it is very unlikely for two signals to appear in a radial velocity data set entrenched so close to such an MMR, down to the level of minutes. Also the peak in the BIS periodogram is not significant, with many more stronger peaks in the data, and since we included correlations between the BIS and the radial velocities, we can be confident that this is not the source of the short period signal we detect. We also note that any power in the activity indicators at a frequency that could give rise to an alias in a periodogram search for signals will not be detected by our Bayesian analysis, since our selection must give rise to signals with amplitudes that are significantly different from zero. This also protects our method from being confused by peaks that we see in the window function. Therefore, we must conclude that the two signals we detect close to a 7:5 MMR 

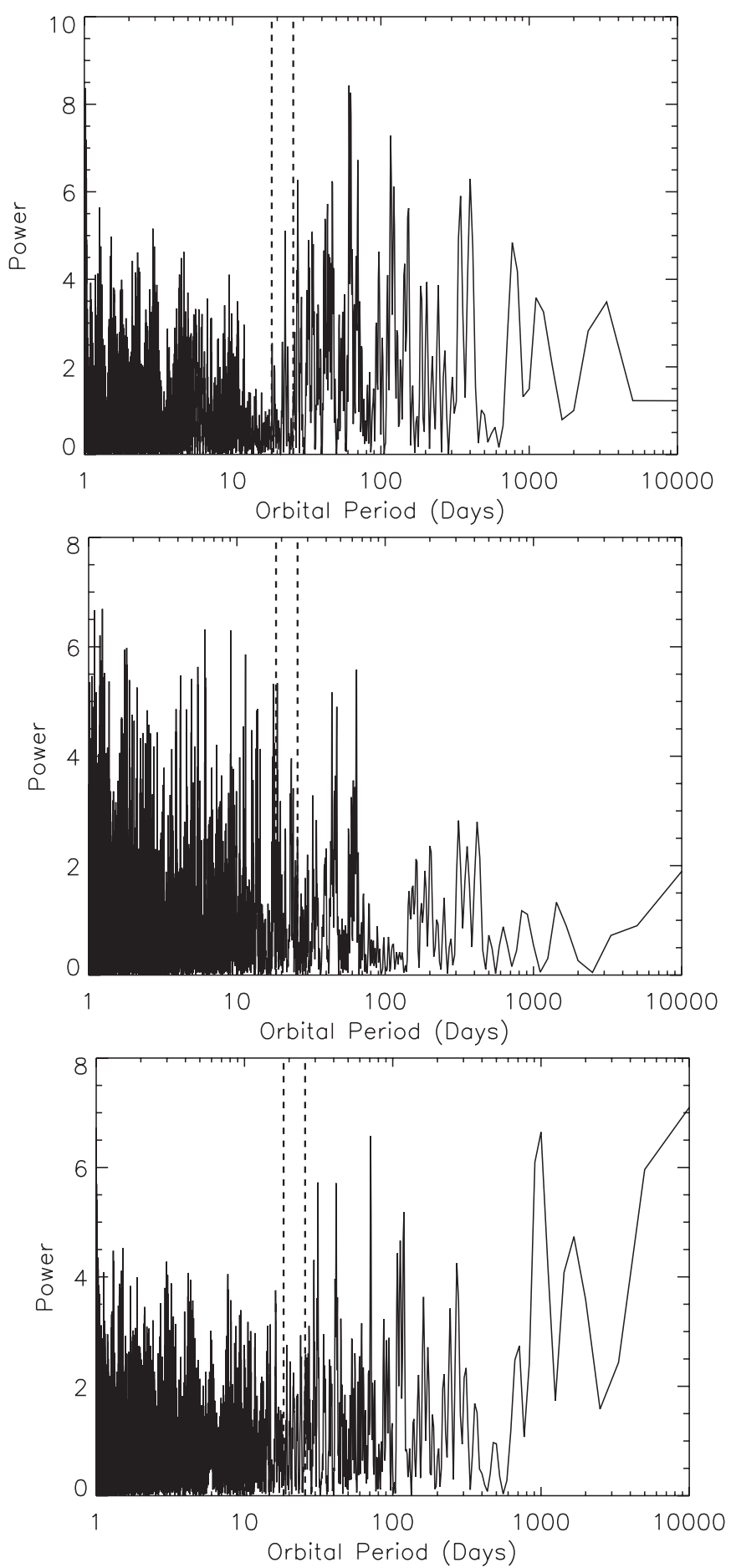

Figure 5. Periodograms of the activity indices we consider. From top to bottom we show the $S$ indices, BIS values, and the FWHM values.

are genuine Doppler signals, induced by the presence of two low-mass planets orbiting HD 41248.

\section{SYSTEM STABILITY}

The HD 41248 system poses an interesting dynamical challenge because of the proximity to a 7:5 MMR and the close spacing of the two planets, both to each other and to the central star. Here general relativistic (GR) effects play a role in the dynamical stability. Before moving on to perform a numerical
Table 6

Chambers (2001) Stability Quantities for the Terrestrial Planets, Giant Planets, and HD 41248

\begin{tabular}{lccrrr}
\hline \hline System & AMD & $S_{m}$ & \multicolumn{1}{c}{$S_{S}$} & \multicolumn{1}{c}{$S_{c}$} & \multicolumn{1}{c}{$S_{H}$} \\
\hline VE & $8 \times 10^{-4}$ & 0.554 & 17.5 & 204.5 & 26.2 \\
JS & $1.6 \times 10^{-3}$ & 0.769 & 8.3 & 80.3 & 7.9 \\
HD 41248 & $3.4 \times 10^{-3}$ & 0.560 & 6.9 & 411.0 & 8.5 \\
\hline
\end{tabular}

search for stable configurations, we list several key properties of the system that determine its stability.

Chambers (2001) defined several measures that he used to quantify a system of planets, which are the angular momentum deficit (AMD; Laskar 1997), the fraction of total mass in the most massive planet $\left(S_{m}\right)$, a spacing parameter $\left(S_{s}\right)$ that scales as the planet to star mass ratio $\mu^{1 / 4}$ rather than the Hill relation of $\mu^{1 / 3}$ (Chambers et al. 1996), and a concentration parameter $\left(S_{c}\right)$ which measures how the mass is concentrated in an annulus. We supplement this with the measure stating the average spacing in Hill radii $\left(S_{H}\right)$. The values of these quantities for Venus and Earth, Jupiter and Saturn, and HD 41248 are listed in Table 6.

As one may see, the AMD of HD 41248 is nearly twice as high as that of Jupiter and Saturn, and much higher than that of Venus and Earth. Systems with higher AMD have the possibility of being more chaotic (Laskar 1997) and have more opportunity to exchange it among the planets. The relatively high AMD of HD 41248 and the fact that one planet appears much more eccentric than the other suggests that both eccentricities will fluctuate with a large amplitude, with one planet being at a minimum (c) when the other is at a maximum (b).

Given that both planets have a nearly equal mass it is no surprise that $S_{d} \sim 0.5$. More interesting is the spacing parameter. This quantity is only $\sim 7$ while for Venus and Earth it is about 18. Even for Jupiter and Saturn it is slightly larger. However, it is interesting to see how $S_{H}$ scales as a function of the masses of the planets. We have $S_{H}=\left(a_{c}-a_{b}\right) / r_{H}$, where $r_{H}$ is the mutual Hill radius, i.e., $r_{h}=\frac{1}{2}\left(a_{b}+a_{c}\right)\left[\left(m_{b}+m_{c}\right) / 3 M_{*}\right]^{1 / 3}$. This can be solved for $a_{b} / a_{c}$ to give

$$
\frac{a_{b}}{a_{c}}=\frac{2 \Gamma-S_{H}}{2 \Gamma+S_{H}},
$$

where $\Gamma=\left[3 M_{*} /\left(m_{b}+m_{c}\right)\right]^{1 / 3}$. Setting $S_{H}=7$ and $m_{b} \sim m_{c} \sim$ $10 M_{\oplus}$ yields $a_{b} / a_{c} \sim 0.83$, but when inserting the masses of Jupiter and Saturn one has $a_{J} / a_{S} \sim 0.58$. Thus, even though Jupiter and Saturn have a similar spacing to HD 41248 in terms of their mutual Hill radii, they are spaced farther apart in relative semi-major axis ratio than the HD 41248 system, implying that Jupiter and Saturn need a higher eccentricity to begin crossing their orbits than the planets of HD 41248. Indeed, for HD 41248 the orbits begin to cross when both planets have an eccentricity near 0.1 , so unless they are apsidally aligned or protected by a resonance, they will become unstable. The nominal eccentricity of planet b is near 0.1 , so the system is close to instability and the stability may crucially depend on their phasing.

In Figure 6 we have plotted the secular evolution of both planets using the nominal orbital elements. The value of $\omega_{c}$ is unconstrained so we set $\Delta \varpi=\omega_{b}-\omega_{c}$, i.e., the difference in the argument of periastron, to $20^{\circ}$. The left panel shows the evolution of planet $b$ in the eccentricity $-\Delta \varpi$ plane while the right panel shows the same for planet c. The planets are in apsidal alignment, i.e., $\Delta \varpi$ librates around 0 and both planets show substantial excursions in their eccentricity amplitude. This alignment prevents many close encounters. We did not find the 

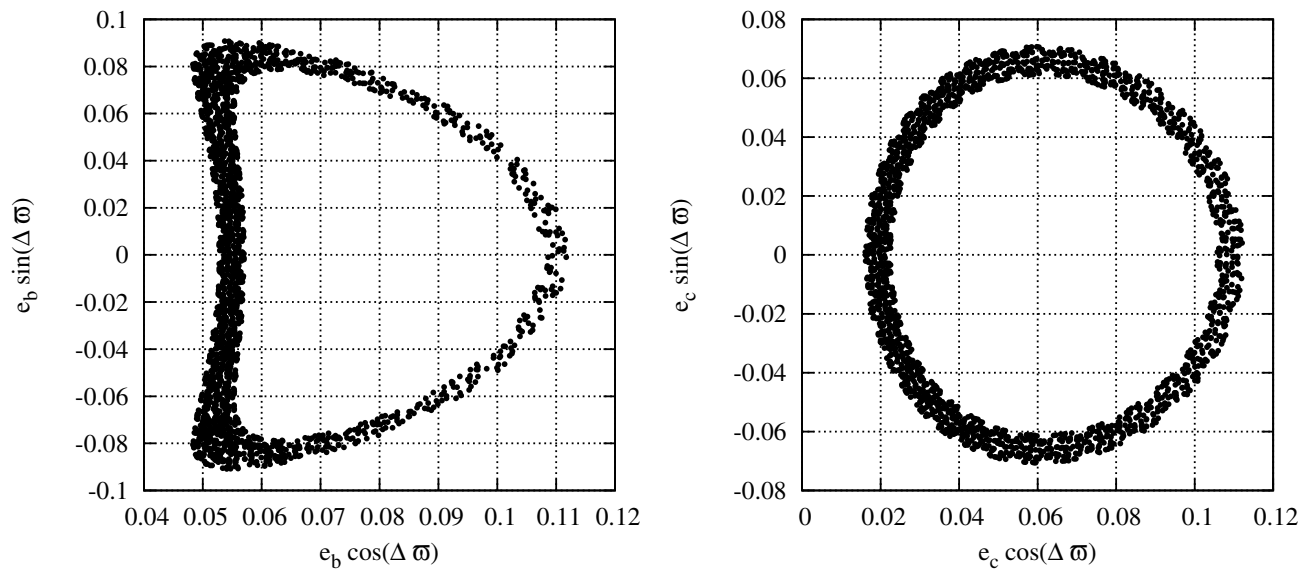

Figure 6. Secular evolution of the two planets of HD 41248. The left panel shows the evolution of planet $\mathrm{b}$ in the $e-\Delta \varpi$ plane while the right panel shows the same for planet $\mathrm{c}$. The planets are in apsidal alignment $(\Delta \varpi$ librates around 0$)$.

resonant angles to librate for this simulation. The question is whether this motion persists for all initial conditions allowed by the observations. Numerical simulations should shed light on this issue.

\subsection{Numerical Methods}

We proceeded to perform a large number $(\sim 40,000)$ of short simulations of the HD 41248 system, which were then analyzed for stability and or chaos.

We performed grid searches of both planets in the $a-\omega$ and $a-e$ planes. The stability dependence upon the mutual inclination was not tested. At this stage, the dependence on the masses was also not tested because of the large possible range depending on the orientation of the system with respect to the observer. However, an estimate of the stability dependence on the masses can be made and maximum masses can be computed as follows.

For a planet on a fairly eccentric orbit, the chaotic boundary surrounding this planet is given by (Mustill \& Wyatt 2012)

$$
\frac{\delta a}{a} \sim 1.8(e \mu)^{1 / 5}
$$

This differs from the $\mu^{2 / 7}$ law of Wisdom (1980) since it takes eccentricity into account. Taking both planets as $10 M_{\oplus}$ and setting their eccentricities as $\sim 0.05$ we have $\delta a / a \sim 0.12$. The planets are spaced $0.035 \mathrm{AU}$ apart and thus $\delta a / a<1-a_{b} / a_{c}$, suggesting that the system could be stable. Chaotic instability would set in when $\delta a / a \sim 1-a_{b} / a_{c}$, which can be solved and yields $e \mu \gtrsim 2 \times 10^{-5}$. Taking $e \sim 0.1$ implies that the masses need to exceed $60 M_{\oplus}$, well outside the uncertainties.

We are dealing with two planets on planar orbits, so that we have four degrees of freedom: the semi-major axis or period ratio, the eccentricities, and $\Delta \varpi$. We performed a grid search for all of these parameters.

The first grid search was done in the semi-major axis argument of the periastron plane of planet $b$, keeping all other orbital elements at their nominal values. The inclination and longitude of the ascending node were set to zero. For planet c, Figure 3 shows that $\omega_{c}$ is almost arbitrary, so we set it to 0 for the sake of simplicity. We varied $\omega_{b}$ evenly in steps of $3^{\circ}$ between $0^{\circ}$ and $360^{\circ}$ and sampled $a_{b}$ assuming a Gaussian distribution with mean and standard deviation given in Figure 3 using 99 steps. This resulted in 11,880 simulations. The sampling method employed here takes into account the density distribution of the semi-major axis but does not consider mutual correlations between the elements.

The planets were integrated for 20,500 yr using the SWIFT MVS integrator (Levison \& Duncan 1994). We included the effects of GR by adding the potential term $V=$ $-3\left(G M_{*} / c\right)^{2} a\left(1-e^{2}\right) / r^{3}$, where $c$ is the speed of light (Nobili $\&$ Will 1986). This term reproduces the GR effect of Mercury's perihelion advancement. For all simulations the time step was set to $2 \times 10^{-4} \mathrm{yr}$ and output was every $10 \mathrm{yr}$.

We analyze the stability of the system using frequency analysis (Laskar 1993). The basic method is as follows: using a numerical integration of the orbit over a time interval of length $T$, we compute a refined determination of the semi-major axes $a_{1}, a_{2}$ obtained over two consecutive time intervals of length $T_{1}=T_{2}=T / 2$. The stability index $D=\max \left(\left|1-a_{2} / a_{1}\right|\right)$ provides a measure of the chaotic diffusion of the trajectory. Low values close to zero correspond to a regular solution, while high values are synonymous with strong chaotic motion (Laskar). The advantage of the frequency analysis method is that it does not require long-term simulations and thus large regions of phase space can be tested with a reasonable amount of CPU power (Correia et al. 2005).

A similar methodology was employed for the other three sets of simulations. The eccentricities were sampled according to the best-fit distributions and we set $\Delta \varpi=10^{\circ}$.

\subsection{Results}

In this section, we present the results from the numerical simulations determining the stable regions. These results will be presented in a series of figures.

Figure 7 displays the stability index of the system as a function of the period ratio $P_{c} / P_{b}$ and $\Delta \varpi$. For this figure we varied the value of $a_{b}$ within its observed range. The axis labels indicate the quantity that was varied while the color coding shows $\log D$. Motion where $\log D \gtrsim 10^{-3}$ is considered chaotic and possible instability may occur (Correia et al. 2005).

An interesting feature is the seven horizontal regions of strong chaos and instability that appear independent of period ratio but occur at regular intervals as $\Delta \varpi$ varies. We verified that in these regions the motion is unstable: the planets encounter each other on short timescales. Varying $a_{c}$ and $\omega_{c}$ results in essentially the same outcome as that displayed in Figure 7. The structure at low $\Delta \varpi$ and low period ratio marks the limits of the 7:5 resonance. 


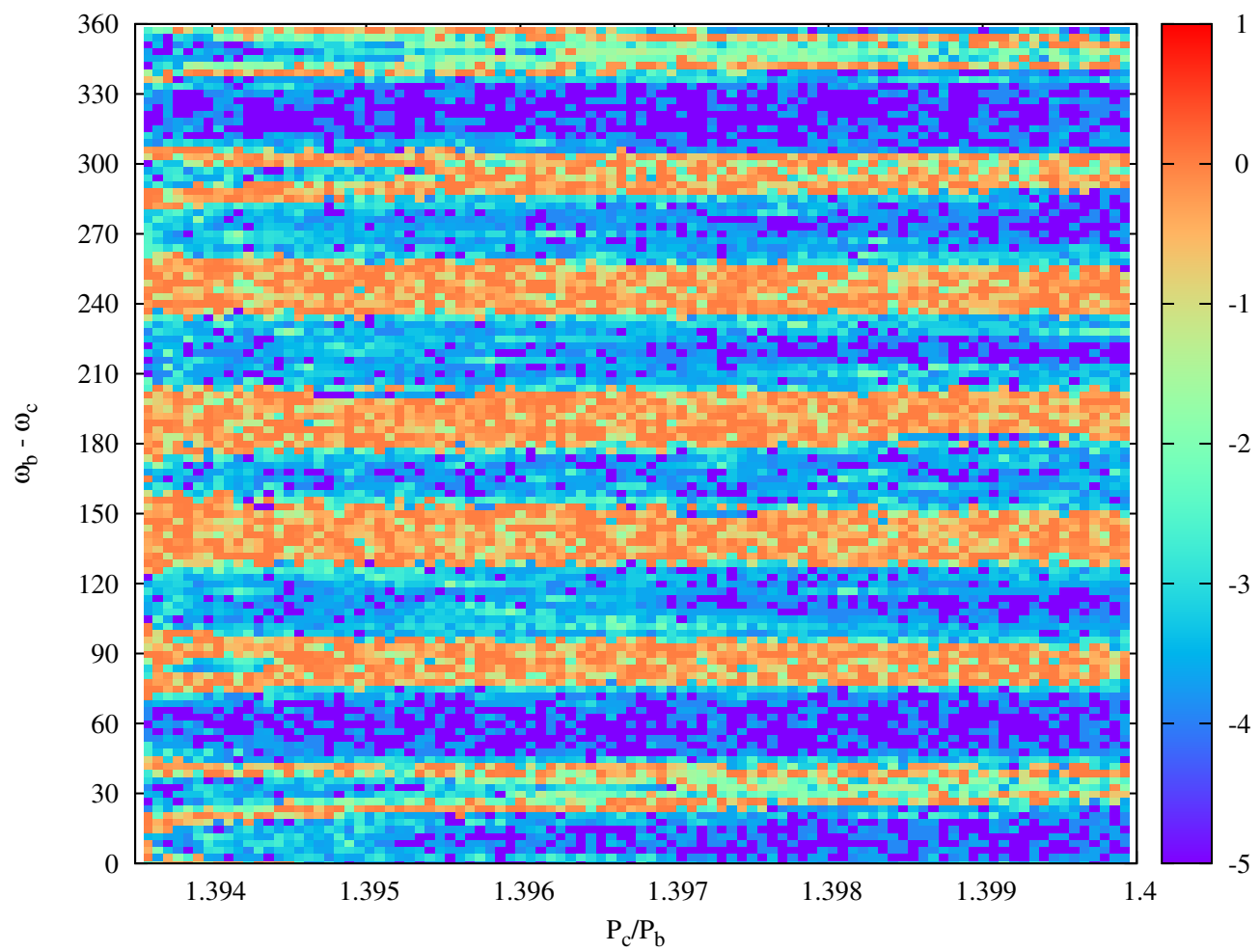

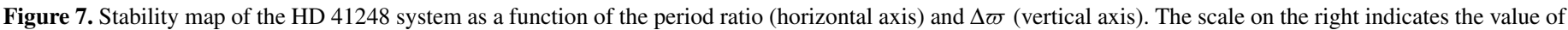
the stability index $D$. The blue regions are stable and the red regions are unstable. We varied $a_{b}$ and $\omega_{b}$.

(A color version of this figure is available in the online journal.)

The reason for these regions of unstable motion has to do with the proximity of the 7:5 resonance. Without the resonance the system would only have a single degree of freedom, $\Delta \varpi$, and thus be integrable (Michtchenko \& Malhotra 2004). The integrable system may exhibit one of three types of motion: $\Delta \varpi$ librates around 0 (apsidal alignment), $\Delta \varpi$ circulates, and $\Delta \varpi$ librates around $180^{\circ}$ (apsidal anti-alignment; see Morbidelli et al. 2009 for a discussion on these three types of motion). Without the presence of the resonance, increasing the initial value of $\Delta \varpi$ from $0^{\circ}$ to $360^{\circ}$ results in the system displaying all three types of motion in three distinct regions.

It should be noted that regions surrounding the period ratio of these planets are clustered with potential MMRs. Nearby and stronger first-order MMRs are the 3:2 and 4:3 commensurabilities, similar in strength to the 6:4 and 8:6 second-order MMRs found around this dynamical region, and so are the weaker 10:7 and 11:8 third-order MMRs. Therefore, we ran some tests to ensure that the 7:5 MMR is the most likely dynamical configuration for this system, given the orbital parameters. Taking the periods and uncertainties from Table 5, and assuming Gaussian distributions for each, we computed the distribution of the period ratio $\left(P_{c} / P_{b}\right)$ and found a mean and $\sigma$ of 1.397 and 0.003 , respectively. The narrow range of periods given by the orbital elements constrains the possible MMR to be only the 7:5 ratio, since even the nearest 10:7 and 11:8 MMRs are found to be $8 \sigma$ and $6 \sigma$ away, respectively.

From the analysis of the motion of $\Delta \varpi$ we find that the system most likely has $\Delta \varpi$ librating around 0 because this configuration yields all stable solutions. When $\Delta \varpi$ circulates or librates around $180^{\circ}$ the system is chaotic and often unstable.

However, the presence of the resonance changes the system's behavior. The 7:5 is second order, and thus there are three resonant arguments:

$$
\begin{aligned}
\sigma_{b} & =5 \lambda_{b}-7 \lambda_{c}+2 \varpi_{b}, \\
\sigma_{c} & =5 \lambda_{b}-7 \lambda_{c}+2 \varpi_{c}, \\
\sigma_{b c} & =5 \lambda_{b}-7 \lambda_{c}+\varpi_{b}+\varpi_{c},
\end{aligned}
$$

where $\lambda=\varpi+M$ is the mean longitude and $\varpi=\Omega+\omega$ and $M$ is the mean anomaly. The system is planar and thus $\Omega$ is undefined (we set it to zero). The proximity of the resonance causes the seven maxima and minima in $D$ as a function $\Delta \varpi$ as it circulates or librates around $180^{\circ}$.

We have decided to display this evolution in a series of figures. Starting from the bottom right corner of Figure 7 the system becomes unstable when $\Delta \varpi \sim 30^{\circ}$ so a transition must occur. We displayed the evolution in the $e_{b}-\Delta \varpi$ (left column) and $e_{c}-\Delta \varpi$ planes (right column) for three simulations in Figure 8. The top panels depict the evolution while the motion is still regular, the middle panels show the motion at the edge of the instability strip, and the bottom panels show the motion for an unstable configuration (although we only depicted the motion until the instability occurred).

In the top panels both planets have $\Delta \varpi$ librate around 0 . Increasing the initial value of $\Delta \varpi$ from the top panels to the middle panels, one sees a fundamental change in the middle left panel. There appear to be two regions of motion: one in which $\Delta \varpi$ librates around 0 and one in which it circulates. The motion starts as libration around 0 with a mean eccentricity of 0.08 and a small amplitude (small loop on the right), but the system encounters a saddle and can librate around 0 with large amplitude changes of the eccentricity. The full motion is libration of $\Delta \varpi$ around 0 but it encompasses two separate regions. However, every once in a while the separatrix is crossed and $\Delta \varpi$ also circulates. This suggests that the system is on the 

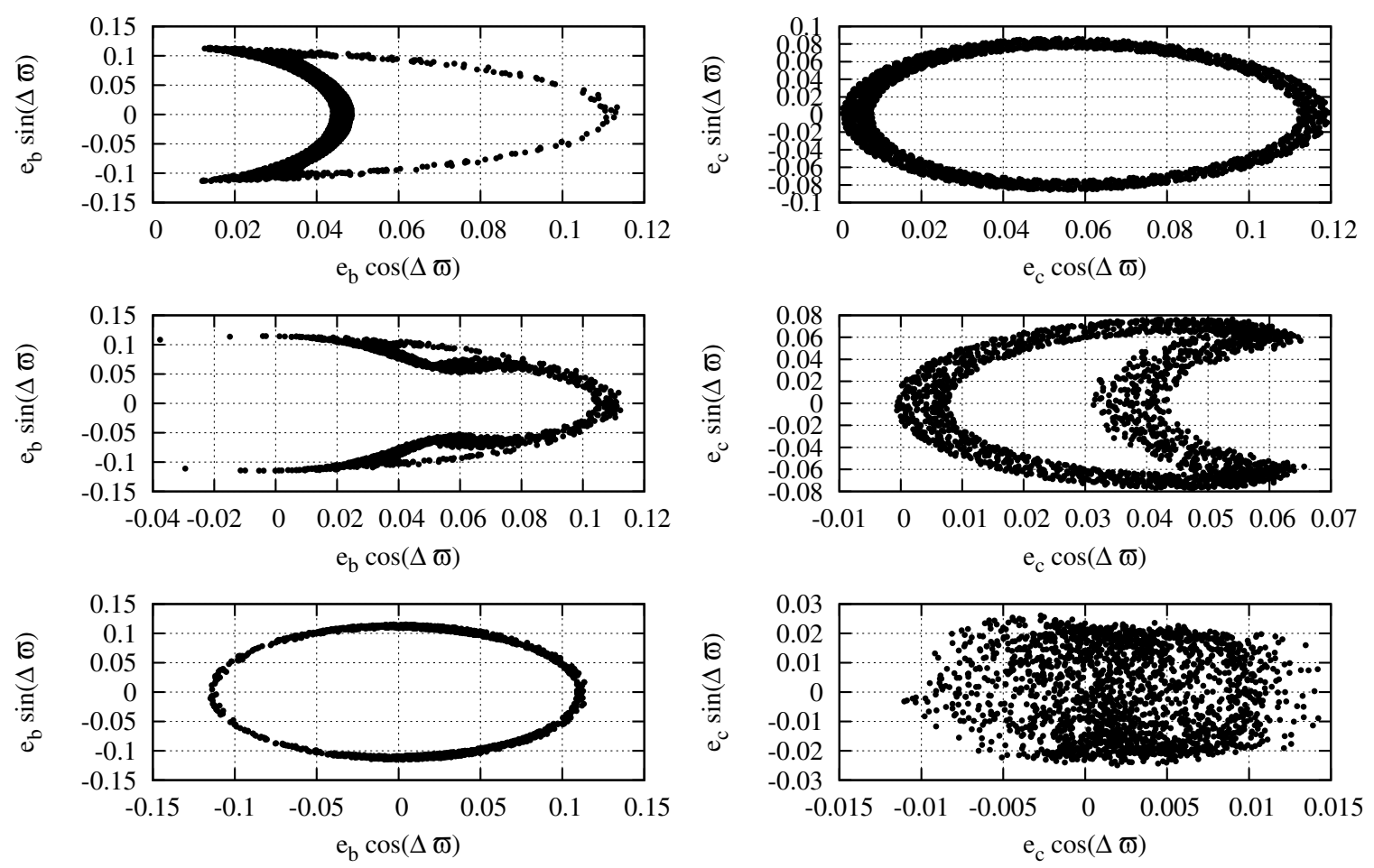

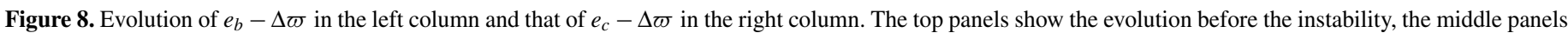
are at the stability edge, and the bottom panels show the evolution for an unstable system.

edge of a resonance. The motion for planet $\mathrm{c}$ has also changed: previously it showed a single loop but now it encompasses a moon-shaped region that is typical for libration in a resonance (e.g., Murray \& Dermott 1999). In the lowest panels $\Delta \varpi$ circulates because the planets have crossed the separatrix. The apsidal no longer prevents them from encounters and the system is unstable.

We searched our solutions for libration of the angles $\sigma$ but we found no case where these angles librated. We checked this by calculating the resonant angles for each simulation and tabulating the mean, minimum, and maximum values. If libration occurs then the minima and maxima should be different from 0 and 360 and the mean should be 0 or 180 . However, in each case we always found the minima and maxima to be 0 and 360 , and the mean to be close to 180 , suggesting that the angles circulate. We have inspected a large sample by eye and conclude that we did not witness any libration occurring. While this procedure should break down for unstable cases, configurations which are stable for the entire simulation duration should have their minima and maxima confined to a small range. We believe this could be caused by the influence of GR, which primarily affects the pericenter precession of close-in planets. In turn, this can help break or support a resonant configuration and also affect the stability of multi-planet systems (e.g., Veras \& Ford 2010).

The stability dependence of the system on the eccentricities of both planets is displayed in Figures 9 and 10. For these simulations we set the initial value of $\Delta \varpi=10^{\circ}$ so that we would not witness any instability related to this quantity. We varied $a_{b}$ and $e_{b}$ for Figure 9 and $a_{c}$ and $e_{c}$ for Figure 10. As one may see, the system is mostly stable as a function of $P_{c} / P_{b}$ and $e_{b}$ unless $e_{b} \gtrsim 0.2$, besides some regions at low period ratio. For such high values of $e_{b}$ the apsidal alignment does not protect the planets from encounters because the orbit of planet $b$ already crosses that of planet $\mathrm{c}$, regardless of the eccentricity of planet $\mathrm{c}$.
There is some fine structure visible in the figure of which the line going from $(1.3955,0.08)$ to $(1.4,0.03)$ is the most interesting. Below this line there is a large region of stable motion and here $\Delta \varpi$ circulates. However, the eccentricities are small enough that the planets do not cross their orbits and they are protected by the 7:5 resonance. Above this region the stability is guaranteed by the apsidal alignment (apart from at high eccentricity).

In Figure 10 we see a large unstable region at low eccentricity and low period ratio. Here the planets are at the edge of the resonance which results in chaotic motion and large excursions in eccentricity resulting in encounters. The stable region on the right has the motion being dominated by small eccentricity and by the resonance so there are few encounters, while at high eccentricity and low period ratio the planets are not in resonance but the apsidal alignment prevents close encounters.

In summary, a system of two super-Earth planets is most likely in the HD 41248 data when one considers the nominal orbits, provided that the apses are nearly aligned. Given that $\omega_{c}$ appears unconstrained by observations, this configuration is a viable outcome. The apsidal alignment provides protection against close encounters at high and low eccentricities, as long as the eccentricities are of comparable value. If the eccentricity of one planet exceeds 0.2 while the other is nearly zero, the system becomes unstable. However, such extreme configurations can be ruled out by the observational uncertainties.

\section{DISCUSSION}

The population of low-mass exoplanets tends to differ significantly from that of more massive planets. Observational evidence for a lack of low-mass planets orbiting the most metalrich stars (Jenkins et al. 2013) points to fundamentally different evolutionary properties between low- and high-mass planets. As discussed in the Introduction, there also appears to be a 


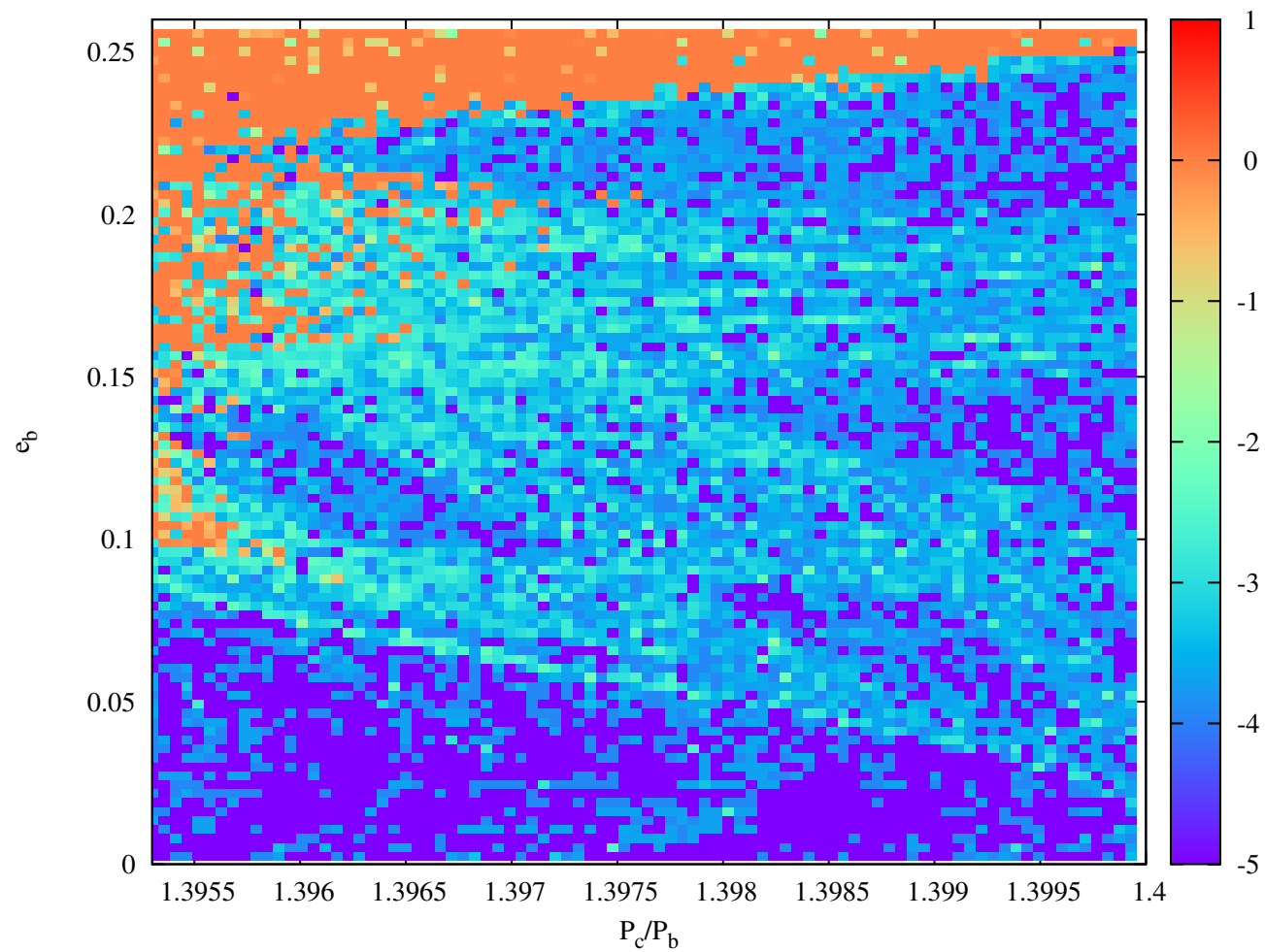

Figure 9. Stability map of the HD 41248 system as a function of the period ratio (horizontal axis) and $e_{b}$ (vertical axis). The scale on the right indicates the value of the stability index $D$. The blue regions are stable and the red regions are unstable. We varied $a_{b}$ and $e_{b}$.

(A color version of this figure is available in the online journal.)

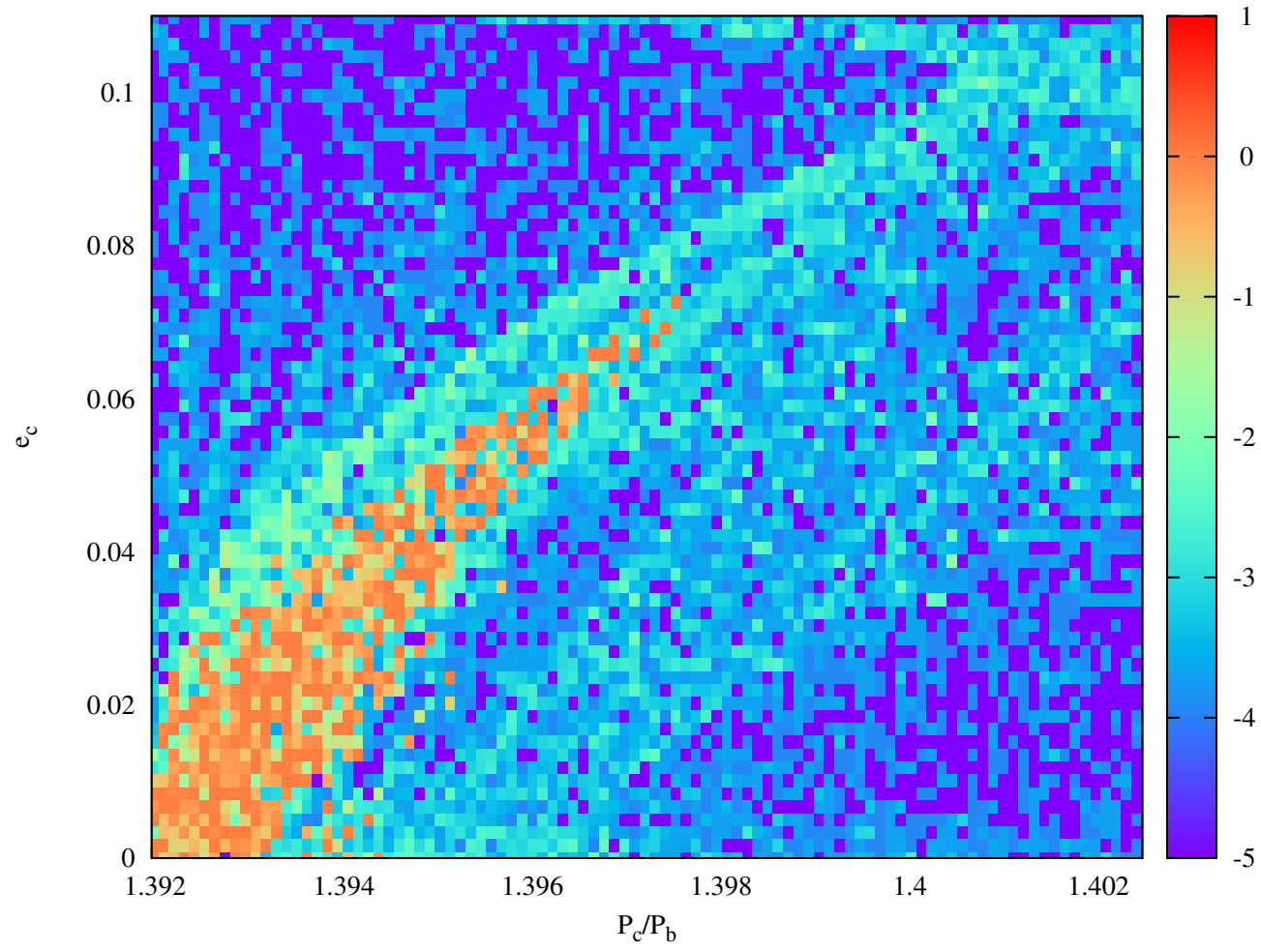

Figure 10. Same as Figure 10, but now we varied $a_{c}$ and $e_{c}$.

(A color version of this figure is available in the online journal.) 
higher fraction of dynamically packed low-mass planetary systems with planets close to MMRs.

Here we report the discovery of a possible 7:5 MMR planetary system, consisting of two super-Earths orbiting a metal-poor star. Alone, this discovery adds to the number of metal-poor stars with low-mass planets and lends more weight to the hypothesis presented in Jenkins et al. It could also be the first confirmed super-Earth system of planets in such a secondorder resonant configuration since libration of the longitude of periastrons yields a highly stabilized system, and our data set and simulations leave the possibility for libration of the resonant angles and hence resonance. Also the Doppler data period ratio differs from the true 7:5 period ratio by less than 1 part in 300, a strong argument in favor of the MMR.

Around one-third of multi-planet systems discovered by radial velocities are found to be close to an MMR (Lissauer et al. 2011), but the fraction actually in the resonance is significantly lower. However, Lissauer et al. show that the number of known systems in an MMR is significantly more than that expected from a randomly drawn population. Predictions from convergent migration show that around $1 \%$ of resonant systems should last for around a disk lifetime (Adams et al. 2008) whereas planet-planet scattering mechanisms yield MMRs around $5 \%-10 \%$ of the time (Raymond et al. 2008). If we take the Kepler resonant numbers from Lissauer et al. at face value, since the number of false positives from multi-transiting systems is expected to be significantly lower than that from single transiting events (Latham et al. 2011), then we might expect that the HD 41248 planetary system was formed through the planet-planet scattering mechanism. However, it may not be so straightforward.

Terquem \& Papaloizou (2007) studied the migration of cores through a protoplanetary disk that includes mutual interactions between the cores and found that the presence of MMRs is common. They found that simulations that began with a larger outer planetary distribution radius, or that started with significantly lower core masses, would tend to produce the 7:5 MMR. This indicates that such a second-order MMR that forms in this way requires longer timescales, since reducing the core masses or increasing the planetary core distance from the star gives rise to a longer evolutionary time, and thus if the HD 41248 planets were formed in this way we might expect that they started with low initial core masses, possibly by forming late in the disk evolution, or they started life much farther out in the disk than their current semi-major axes, or most likely a combination of both low core masses and long convergent migration. It is interesting to note that even though planet-planet scattering simulations produce more MMRs, the results from Raymond et al. (2008) never resulted in a 7:5 MMR commensurability.

Finally, we should note the mass ratio between these planets. HD $41248 \mathrm{~b}$ is found to be $12.3 M_{\oplus}$ and planet c is $8.6 M_{\oplus}$, giving rise to a mass ratio of 0.7 . The simulations of Terquem \& Papaloizou (2007) ended with much lower mass ratios for the planets that were found in the 7:5 configuration. They found mass ratios of 0.1 and 0.2 ; however, the more massive planet was the one closer to the star, similar to the system we present here. We note that finding MMRs that have much lower mass ratios is difficult due to the inherent difficulties of first discovering very low-amplitude signals in radial velocity data sets, and also the difficulty of finding low-amplitude multi-planet signals when the super-position of the Keplerians has a strong dominating signal and a much weaker and longer period signal. With this in mind, we expect more of these systems to emerge from the radial velocity data with the addition of more Doppler data and better analysis techniques.

We thank the anonymous referee and the editor, Eric Feigelson, for helpful comments that made this a better manuscript. We also thank Guillem Anglada-Escudé for providing us with the HARPS-TERRA code. J.S.J. also acknowledges funding by Fondecyt through grant 3110004 and partial support from CATA (PB06, Conicyt), the GEMINI-CONICYT FUND, and from the Comité Mixto ESO-GOBIERNO DE CHILE. This research has made use of the SIMBAD database and the VizieR catalogue access tool, operated at CDS, Strasbourg, France.

\section{REFERENCES}

Adams, F. C., Laughlin, G., \& Bloch, A. M. 2008, ApJ, 683, 1117

Anglada-Escudé, G., Arriagada, P., Vogt, S. S., et al. 2012, ApJL, 751, L16

Anglada-Escudé, G., \& Butler, R. P. 2012, ApJS, 200, 15

Anglada-Escudé, G., \& Tuomi, M. 2012, A\&A, 548, A58

Baluev, R. V. 2013, MNRAS, 429, 2052

Buchhave, L. A., Latham, D. W., Johansen, A., et al. 2012, Natur, 486, 375 Casagrande, L., Schönrich, R., Asplund, M., et al. 2011, A\&A, 530, A138 Chambers, J. E. 2001, Icar, 152, 205

Chambers, J. E., Wetherill, G. W., \& Boss, A. P. 1996, Icar, 119, 261

Correia, A. C. M., Udry, S., Mayor, M., et al. 2005, A\&A, 440, 751

Desort, M., Lagrange, A.-M., Galland, F., et al. 2008, A\&A, 491, 883

Fabrycky, D. C., Ford, E. B., Steffen, J. H., et al. 2012, ApJ, 750, 114

Fischer, D. A., \& Valenti, J. 2005, ApJ, 622, 1102

Gomes, R., Levison, H. F., Tsiganis, K., \& Morbidelli, A. 2005, Natur, 435,466

Haario, H., Saksman, E., \& Tamminen, J. 2001, Bernoulli, 7, 223

Jenkins, J. S., Jones, H. R. A., Pavlenko, Y., et al. 2008, A\&A, 485, 571

Jenkins, J. S., Jones, H. R. A., Tinney, C. G., et al. 2006, MNRAS, 372, 163

Jenkins, J. S., Jones, H. R. A., Tuomi, M., et al. 2013, ApJ, 766, 67

Jenkins, J. S., Murgas, F., Rojo, P., et al. 2011, A\&A, 531, A8

Laskar, J. 1993, CeMDA, 56, 191

Laskar, J. 1997, A\&A, 317, L75

Laskar, J., \& Correia, A. C. M. 2009, A\&A, 496, L5

Latham, D. W., Rowe, J. F., Quinn, S. N., et al. 2011, ApJL, 732, L24

Levison, H. F., \& Duncan, M. J. 1994, Icar, 108, 18

Lissauer, J. J., Ragozzine, D., Fabrycky, D. C., et al. 2011, ApJS, 197, 8

Marcy, G. W., Butler, R. P., Fischer, D., et al. 2001, ApJ, 556, 296

Michtchenko, T. A., \& Ferraz-Mello, S. 2001, AJ, 122, 474

Michtchenko, T. A., \& Malhotra, R. 2004, Icar, 168, 237

Morbidelli, A., Brasser, R., Tsiganis, K., Gomes, R., \& Levison, H. F. 2009, A\&A, 507, 1041

Morbidelli, A., Levison, H. F., Tsiganis, K., \& Gomes, R. 2005, Natur, 435, 462 Murray, C. D., \& Dermott, S. F. 1999, Solar System Dynamics (Cambridge: Cambridge Univ. Press)

Mustill, A. J., \& Wyatt, M. C. 2012, MNRAS, 419, 3074

Nobili, A. M., \& Will, C. M. 1986, Natur, 320, 39

Pavlenko, Y. V., Jenkins, J. S., Jones, H. R. A., Ivanyuk, O., \& Pinfield, D. J. 2012, MNRAS, 422, 542

Peale, S. J. 1976, ARA\&A, 14, 215

Perryman, M. A. C., Lindegren, L., Kovalevsky, J., et al. 1997, A\&A, 323, L49

Queloz, D., Henry, G. W., Sivan, J. P., et al. 2001, A\&A, 379, 279

Raymond, S. N., Barnes, R., Armitage, P. J., \& Gorelick, N. 2008, ApJL, 687, L107

Rivera, E. J., Laughlin, G., Butler, R. P., et al. 2010, ApJ, 719, 890

Santos, N. C., Gomes da Silva, J., Lovis, C., \& Melo, C. 2010, A\&A, 511, A54 Sousa, S. G., Santos, N. C., Israelian, G., Mayor, M., \& Udry, S. 2011, A\&A, 533, A141

Terquem, C., \& Papaloizou, J. C. B. 2007, ApJ, 654, 1110

Tsiganis, K., Gomes, R., Morbidelli, A., \& Levison, H. F. 2005, Natur, 435, 459 Tuomi, M. 2011, A\&A, 528, L5

Tuomi, M. 2012, A\&A, 543, A52

Tuomi, M., Anglada-Escudé, G., Gerlach, E., et al. 2013a, A\&A, 549, A48

Tuomi, M., \& Jenkins, J. S. 2012, arXiv e-prints

Tuomi, M., \& Jones, H. R. A. 2012, A\&A, 544, A116

Tuomi, M., Jones, H. R. A., Jenkins, J. S., et al. 2013b, A\&A, 551, A79

van Leeuwen, F. 2007, A\&A, 474, 653

Veras, D., \& Ford, E. B. 2010, ApJ, 715, 803

Wisdom, J. 1980, AJ, 85, 1122 